University of Zurich

Department of Economics

Working Paper Series

ISSN 1664-7041 (print)

ISSN 1664-705X (online)

Working Paper No. 224

\title{
The Influences of Social Context on the Measurement of Distributional Preferences
}

Matthias Greiff, Kurt A. Ackermann and Ryan O. Murphy

April 2016 


\title{
The influences of social context on the measurement of distributional preferences
}

\author{
Matthias Greiff*
}

Kurt A. Ackermann ${ }^{\dagger}$

Ryan O. Murphy

Monday $29^{\text {th }}$ February, 2016

\begin{abstract}
Different social contexts have been used when measuring distributional preferences. This could be problematic as contextual variance may inadvertently muddle the measurement process. We use a within-subjects design and measure distributional preferences in resource allocation tasks with role certainty, role uncertainty, decomposed games, and matrix games. Results show that, at the aggregate level, role uncertainty and decomposed games lead to higher degrees of prosociality when compared to role certainty. At the individual level, we observe considerable differences in behavior across the social contexts, indicating that the majority of people are sensitive to these different social settings but respond in different ways.
\end{abstract}

Keywords: Distributional Preferences, Social Preferences, Other Regarding Preferences, Social Value Orientation (SVO), Measurement Methods, Individual Differences

JEL: C91, D03, D64

*Justus-Liebig-University Giessen, Department of Economics, VWL VI, Licher Str. 66, 35394 Giessen, Germany, matthias.greiff@wirtschaft.uni-giessen.de

${ }^{\dagger}$ Fehr Advice \& Partners, Klausstrasse 20, 8008 Zürich, Switzerland, kurt.alexander.ackermann@ gmail.com

${ }^{\ddagger}$ University of Zürich, Department of Economics, Blümlisalpstrasse 10, 8006 Zürich, Switzerland, ryan.murphy@econ.uzh.ch 


\section{Introduction: Measuring distributional preferences}

The measurement of distributional preferences is an important issue in economics and psychology (see e.g., Camerer and Fehr, 2004; Fisman et al, 2007; Balliet et al, 2009; Murphy and Ackermann, 2014; Kerschbamer, 2015a), but different measurement methods are used. Each method has two distinct components. First, there are choice sets: well-defined sets of options that correspond to different allocations. The second component is the social context in which a decision maker (DM) makes her choices. Typically, social features of the decision task are minimized, but there remains some minimal social context, as there must be some "other" who is affected by the choices of the DM. Social contexts differ by role assignment and can be accentuated by the display of information.

The design of our experiment is similar to Blanco et al (2011) and Dariel and Nikiforakis (2014). Both use a within-subject design to compare behavior across different games. Instead of comparing behavior across games, we compare subjects' social value orientation (SVO) across different social contexts, broadly considered. Differences in social context are potentially problematic, as they may interact with other social preferences apart from pure distributional preferences, for instance, preferences for reciprocity (Kerschbamer, 2015b). At the aggregate level, we compare the average degree of prosociality and, at the individual level, we compare the consistency of subjects' revealed preferences.

\section{Procedural differences}

\subsection{Different sets of stimuli and the slider measure}

The first component for the measurement of distributional preferences is a resource allocation task. The simplest task of this kind is a dictator game. Other measurements of distributional preferences are based on multiple resource allocation tasks that have a more nuanced structure (e.g., various marginal rates of substitution, see Murphy and Ackermann, 2014, and Kerschbamer, 2013, for surveys comparing different methods). In each task the DM chooses her most preferred option, and, based on her choices, her distributional preferences are revealed.

In this study, we use the SVO Slider Measure which has demonstrable psychometric benefits over alternative measures, including its production of a continuous score and a built-in transitivity check. SVO has been shown to be highly stable as an individual difference with a test-retest reliability of $r=0.915$ (Murphy et al, 2011).

The Slider Measure consists of six resource allocation tasks (see Figure 5 in the Appendix and Murphy et al, 2011). Based on the DM's choices, her SVO is computed as

$$
S V O^{\circ}=\arctan \left(\frac{\bar{\pi}_{o}-50}{\bar{\pi}_{s}-50}\right)
$$

where $\bar{\pi}_{s}$ and $\bar{\pi}_{o}$ denote the DM's and the other's mean payoff. The SVO angle can range between $-16.26^{\circ}$ and $61.39^{\circ}$, while negative angular degrees indicate negative concerns for others, angular degrees close to zero indicate narrow self-interest, and positive angular degrees indicate positive concerns for others. ${ }^{1}$

\footnotetext{
${ }^{1}$ Self-interest does not result in a score of exactly 0 because allocation task 1 is structured such that the DM's
} 


\subsection{Different social contexts}

One way to conceptualize a resource allocation task is to consider a decision situation with two distinct roles, an active Decider and a passive Receiver. The Decider chooses an allocation which determines her own and the Receiver's payoff. This is not a proper game as only the Decider makes a choice. However, it is a social decision as the Decider's choice has an effect on some other person.

There has been substantial contextual heterogeneity in how resource allocation tasks have been implemented. This heterogeneity stems from differences in role assignment, on which we elaborate next.

\subsubsection{Role certainty}

With fixed role assignment or role certainty, subject A is in the role of the Decider and subject B is in the role of the Receiver. B is passive, A knows this, and all of these features are common knowledge. In experimental practice, the sample is split in half and the role of the Decider is assigned to subjects in one half, while the role of the Receiver is assigned to subjects in the other half of the sample. Role certainty has been employed in several experiments (e.g., Forsythe et al, 1994; Dana et al, 2007; Bardsley, 2007).

A downside of this procedure is inefficiency in that the distributional preferences of only half of the subjects are assessed. Another consequence is that this context creates a substantial imbalance in power that is obvious to Decider and Receiver. This imbalance may evoke particular feelings of responsibility or entitlement on behalf of some Deciders, whereas others may not be affected by this imbalance.

\subsubsection{Role uncertainty}

A procedure that avoids the inefficiency of only measuring half the sample is role uncertainty. All subjects perform the resource allocation task but do not know a priori whether they are going to be assigned the role of Decider or Receiver. Only after decisions are made, subjects are matched randomly in pairs and another random process determines which role is assigned to which subject in each pair. Role uncertainty has been employed in several experiments (e.g., Charness and Grosskopf, 2001; Engelmann and Strobel, 2004).

Role uncertainty also mitigates the issue of power imbalance and preserves symmetry. With role uncertainty, all subjects are equally entitled because all subjects perform the same task (Engelmann and Strobel, 2004, 859).

\subsubsection{Double role assignment}

With double role assignment, all subjects simultaneously perform the resource allocation task. A subject's total payoff comes from two sources: the payoff from her role as Decider, and her payoff from her role as Receiver. The mapping from choices to payoffs is common knowledge, and, because the two subjects are interdependent, this is a strategic setting. Double role assignment with random pairs is used in many economic experiments (e.g., Andreoni and Miller, 2002; Fisman et al,

payoff is invariant at 85 and the DM chooses a payoff for the other ranging between 15 and 85 . 
2007; Balafoutas et al, 2012, 2014). Double role assignment with fixed pairs has been employed in several experiments, mostly in psychology where it is most frequently used in the measurement of SVO (e.g., Pruitt, 1967; Messick and McClintock, 1968; Liebrand, 1984; Offerman et al, 1996; van Lange et al, 2007).

Each subject simultaneously gives and receives, so that a subject's decision to give might depend on her expectations about what she will receive from the other. Measuring distributional preferences with double role assignment may yield a confound as expectations may inform choices as well as preferences. Along these lines Rigdon and Levine (2009) find significant differences in choice behavior contingent upon subjects' expectations.

Given double role assignment, there are two ways of displaying information. If information is displayed in a payoff matrix, strategic interdependence is salient because a subject can see clearly that there is another subject whose decision affects their own payoff. Matrix games have not been used to measure social preferences because of this obvious strategic interdependence.

One attempt to control for strategic interdependence are decomposed games, which manipulate how information about payoffs is displayed (Pruitt, 1967; Messick and McClintock, 1968). Assume that $\mathrm{A}$ and $\mathrm{B}$ simultaneously choose between $X=(100,50)$ and $Y=(85,85)$. With the decomposed games method each subject sees both allocations and is informed that the other subject chooses between the same allocations. The normal form representation and the corresponding decomposed game can be seen in Figure 1.

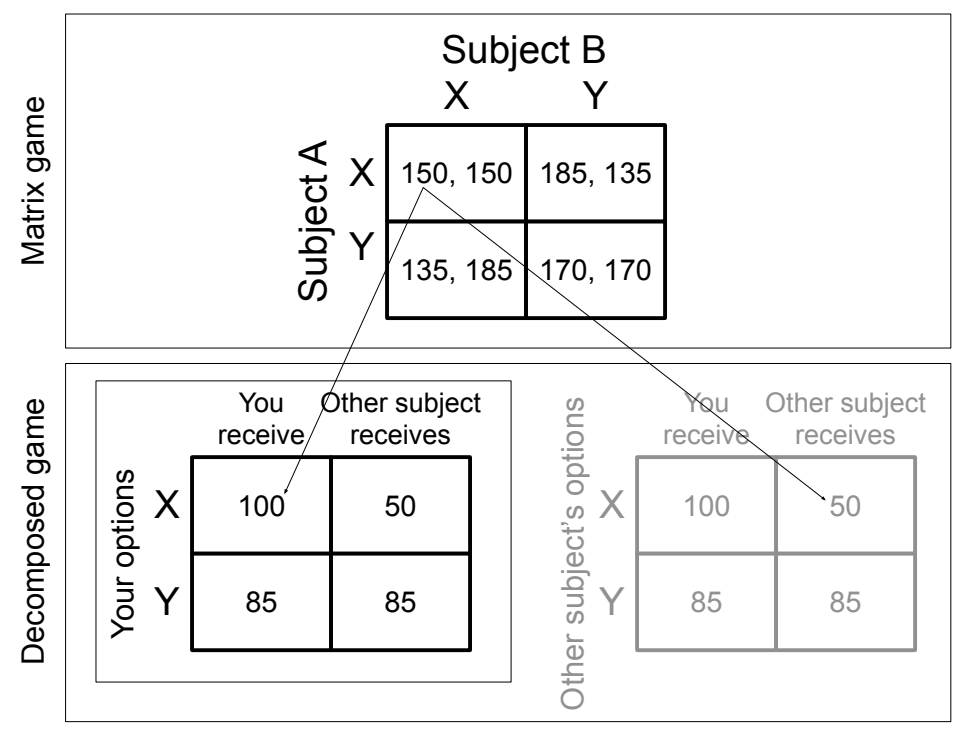

Figure 1: Decomposed game and matrix game. In the matrix game subject A chooses a row and subject B simultaneously chooses a column. In the decomposed game, both subjects simultaneously choose an allocation and the final payoffs are given by the sum of the allocations.

The use of decomposed games may be a misguided experimental manipulation as it does nothing to really mitigate strategic interdependence (see also Greiff, 2013). Decomposed games are still strategic games, but merely designed to be obtuse and thus harder for subjects to understand. Obfuscation is not the same as experimental control, and experimenters may not be able to blur away strategic interdependence. 
A charitable interpretation of the decomposed games approach is that it acts like choice framing, directing a subject's attention to the consequences of her own action while downplaying the potential consequences from the other's choice (Pruitt, 1967).

\section{Hypotheses}

Before we derive the hypotheses we introduce the experimental design (see Figure 2), because understanding the design helps make the hypotheses clearer. There are three different role assignment procedures and one of the procedures has two ways of displaying information. This yields four treatments: the Role Certainty Treatment (RCT), the Role Uncertainty Treatment (RUT), the Decomposed Game Treatment (DGT), and the Matrix Game Treatment (MGT). We use a fully within-subject design with random orderings of treatments to mitigate potential carryover effects. Using this design, we test the following hypotheses.

\begin{tabular}{|c|c|c|c|c|c|c|c|c|c|c|c|c|}
\hline & \multicolumn{6}{|c|}{ individual decision making task } & \multicolumn{6}{|c|}{ strategic interaction (proper game) } \\
\hline treatment & \multicolumn{3}{|c|}{$\begin{array}{l}\text { role certainty } \\
\text { treatment }(\mathrm{RCT})\end{array}$} & \multicolumn{3}{|c|}{$\begin{array}{l}\text { role uncertainty } \\
\text { treatment (RUT) }\end{array}$} & \multicolumn{3}{|c|}{$\begin{array}{l}\text { decomposed game } \\
\text { treatment (DGT) }\end{array}$} & \multicolumn{3}{|c|}{$\begin{array}{c}\text { matrix game } \\
\text { treatment (MGT) }\end{array}$} \\
\hline $\begin{array}{c}\text { role } \\
\text { assignment }\end{array}$ & \multicolumn{3}{|c|}{ fixed role assignment } & \multicolumn{3}{|c|}{ role uncertainty } & \multicolumn{3}{|c|}{ double role assignment } & \multicolumn{3}{|c|}{ double role assignment } \\
\hline \multirow{4}{*}{$\begin{array}{l}\text { simplified } \\
\text { stimulus } \\
\text { example }\end{array}$} & \multicolumn{3}{|c|}{ Options } & \multicolumn{3}{|c|}{ Options } & \multicolumn{3}{|c|}{ Options } & \multicolumn{3}{|c|}{ The other's options } \\
\hline & \multicolumn{3}{|c|}{ A } & \multicolumn{3}{|c|}{ A } & & A & B & \multirow{3}{*}{$\begin{array}{c}\text { Your } \\
\text { options } \\
\text { B }\end{array}$} & A & в \\
\hline & You receive & 100 & 85 & You receive & 100 & 85 & You receive & 100 & 85 & & \begin{tabular}{|l|l|}
150,150 \\
\end{tabular} & 185,135 \\
\hline & $\begin{array}{l}\text { The other } \\
\text { receives }\end{array}$ & 50 & 85 & $\begin{array}{l}\text { The other } \\
\text { receives }\end{array}$ & 50 & 85 & $\begin{array}{l}\text { The other } \\
\text { receives }\end{array}$ & 50 & 85 & & 135, 185 & 170,170 \\
\hline inter & $\downarrow$ & & & & $\begin{array}{l}\text { Chance } \\
\text { evernt }\end{array}$ & & & & & & & \\
\hline
\end{tabular}

Figure 2: Experimental design. The figures in the last row show how influence propagates from a subject to affect her own payoff and the payoff of another subject.

\section{H1: Incentives reduce distributional preferences.}

In the RCT, half of the subjects filled out the Slider Measure with incentives knowing they were the Deciders. The other half filled out the Slider Measure knowing they were the Receivers and that their responses would not have any effect on themselves nor any other subject.

This implementation yields a test of the effect of incentives on distributional preferences. Similar tests have been conducted before. Mentzakis and Mestelman (2013) found no significant effects, but in Forsythe et al (1994), subjects are found to be more generous if decisions are hypothetical.

To see how financial incentives affect behavior, we compare SVO scores within the RCT, comparing subjects who made incentivized choices with subjects who made hypothetical choices. Although previous findings are mixed, we expect that incentivized choices will decrease SVO. 
H2: Role certainty reduces distributional preferences relative to role uncertainty.

Iriberri and Rey-Biel (2011) compare behavior in modified dictator games with role certainty and role uncertainty and find that subjects are more generous with role uncertainty. This could be driven by fairness considerations and/or by the cost of expressing generosity.

Fairness considerations could lead to more prosocial choices in the RUT. Assume that the choice of an allocation that would result in a high payoff for the other subject is perceived as nice. If $\mathrm{A}$ has a preference for fairness and expects $\mathrm{B}$ to choose the nice allocation, A might reciprocate by also choosing the nice allocation (Rabin, 1993). Here, A's choice depends on her expectation about B's intention.

Higher generosity with role uncertainty could be due to the low costs of expressing generosity (see also the literature on low cost expressive voting, e.g., Kirchgässner, 1992; Hillman, 2010). Assume that being generous increases utility because subjects derive utility from expressing their generosity (Hillman, 2010). With role certainty, the additional utility from being generous comes with a reduction in own payoff. With role uncertainty, being generous in the role of Decider reduces the expected payoff; the actual payoff is reduced only if the subject is assigned to the role of Decider. Hence, the expected price of expressing generosity is lower in RUT. Due to fairness considerations and the low costs of expressing generosity, we expect subjects to be more generous with role uncertainty.

H3: Strategic interdependence increases distributional preferences.

Shafir and Tversky (1992) report results of an experiment where $25 \%$ of choices from subjects playing sequential and simultaneous prisoner's dilemmas had the following pattern: Subjects chose to defect when they knew the other subject had chosen to defect, they chose to defect when they knew the other subject had chosen to cooperate, yet they chose to cooperate when they did not know the other subject's choice. A possible explanation is that in simultaneous games, subjects cooperate because they are motivated to maximize the sum of payoffs (Shafir and Tversky, 1992, 457). In the sequential prisoner's dilemma, the second mover has control over both outcomes once the first mover has made her choice. This undermines team reasoning and subjects tend to choose the alternative that maximizes their own payoffs (Shafir and Tversky, 1992, 457).

A similar kind of team reasoning could be at work in DGT and MGT. If this is the case, subjects would choose prosocial allocations in treatments DGT and MGT, compared to the treatments RCT and RUT.

H4: Decomposed games increase distributional preferences relative to matrix games.

Previous empirical results show that behavior in decomposed games and strategically equivalent matrix games are different (Pruitt, 1967; Gallo Jr. et al, 1969). For most decompositions, cooperation rates where higher. Analogously, we expect that SVO will be higher in DGT.

H5: Behavior in decomposed games is more similar to behavior in matrix games than to behavior in resource allocation tasks with role certainty.

Our four treatments correspond to four different measurement methods. The correlations among the methods can be treated as a method similarity matrix, which itself can be the basis for further analysis using classical multi-dimensional scaling. This kind of analysis provides evidence into the "distance" among the different methods. Moreover, if the different methods were inconsequential to behavior (i.e., if they all measure the same thing), then the correlations between the treatments would be very high and approximate test-retest correlation levels.

In contrast to hypotheses 1 to 4, this hypothesis is concerned with the consistency of individual behavior. This analysis is useful in that it is sensitive to heterogeneity in responses. Say for 
example that half of the subjects became more prosocial in a particular treatment and half become less prosocial in the same treatment (compared to a baseline treatment). This result would yield a consistent mean level of prosociality, but it would not indicate that overall subjects were behaving consistently.

\section{Experimental procedure}

The experiment was conducted using zTree (Fischbacher, 2007). A total of 192 subjects participated. The order in which subjects encountered the four treatments was fully counterbalanced across sessions with eight subjects per session. ${ }^{2}$

In each treatment subjects are matched pairwise and all subjects decide in six different decision situations. In RCT, RUT and DGT the six situations correspond to the six resource allocation tasks which comprise the items of the SVO Slider Measure (Figure 5). In MGT the six decision situations correspond to six matrix games. Hence, we can compute a subject's SVO for each treatment. Roleassignment is treatment specific, as described in Figure 2.

\section{Results}

Each of the 192 subjects participated in all four treatments. In RCT only half of decisions were incentivized. If not explicitly stated otherwise, only incentivized decisions are used for statistical analysis.

\subsection{Descriptive analysis}

The observed preference distributions are consistent with data from earlier experiments in which SVO was measured (Murphy et al, 2011). In all treatments the most common SVO score is 7.82, corresponding to the maximization of the own payoff. Table 1 summarizes the data on subjects' SVOs. The first four columns contain the descriptive statistics for incentivized choices. In RCT half of the subjects made hypothetical decisions which are summarized in column 6. Pooled observations from RCT are summarized in column 5.

\begin{tabular}{ccccc|cc}
\hline \hline & RCT(inc.) & RUT & DGT & MGT & RCT(all) & RCT(hyp.) \\
\hline$n$ & 96 & 192 & 192 & 192 & 192 & 96 \\
Mean & 16.46 & 17.98 & 17.30 & 18.83 & 17.98 & 19.51 \\
Median & 11.36 & 16.26 & 14.94 & 16.26 & 14.62 & 16.73 \\
Std & 13.06 & 14.40 & 13.97 & 16.92 & 14.74 & 16.18 \\
\hline \hline
\end{tabular}

Table 1: Descriptive analysis on subjects' SVOs.

The scatterplots (Figure 3) and the corresponding rank correlations reveal that there is substantial heterogeneity at the individual level. Each scatterplot compares two treatments and reports

\footnotetext{
${ }^{2}$ For details about the experimental procedure, see Section A in the Appendix.
} 
the corresponding correlation. We see that for all pairwise comparisons, there are data points both above and below the 45-degree line. This indicates changes in distributional preferences at the individual level. There are only 16 out of 192 subjects for whom SVOs were identical in all treatments. ${ }^{3}$ For a total of 37 subjects, SVOs do not differ by more than $5^{\circ}$ between treatments.

\subsection{Hypotheses tests}

Result 1: In RCT there is no significant difference between incentivized and hypothetical decisions (Mann-Whitney test, $p=0.140){ }^{4}$

Although we do not find a difference in generosity, financial incentives significantly reduce variance (Levene test, $p=0.004$ ). If incentivized decisions have less variance because subjects are less likely to respond haphazardly, incentives may be an effective way to reduce noise.

Result 2: On average, we find that subjects in RUT are more prosocial than in $R C T$ (t-test, $p=0.029)$.

Our experiment cannot tell whether this result is driven by fairness considerations or by the cost of expressing generosity. However, if SVO is measured with role uncertainty, a subject's SVO should be considered as an upper bound.

Result 3: SVO does not differ significantly between treatments (RCT,RUT) and (DGT,MGT).

To test for this effect, we computed each subject's average SVO from treatments (RCT,RUT) and (DGT,MGT). Call these averages $S V O$ (nonstr.) and $S V O($ str. $)$. The averages are $S V O($ nonstr. $)=$ 17.97 and $S V O(s t r)=$.19.80 . A signed rank test shows that the median of differences is not significantly different from zero $(p=0.080)$.

Result 4: On average, SVO is not significantly different between DGT and MGT (signed rank test, $p=0.295$ ).

At the aggregate level, there is no difference in SVO. At the individual level, however, the correlation between both treatments is only 0.409, and the variance is significantly higher in MGT (Levene test, $p=0.001$ ). This implies that the majority of subjects are sensitive to the strategic framing, but respond in different ways.

Possibly, the decomposed games framing sufficiently "hides" the other subject so that strategic considerations are less salient in DGT, while the payoff matrix increases the salience of the strategic considerations. With strategic considerations being more salient, choice behavior could be driven by expectations, and individual differences in expectations, which are amplified by the salience of interdependence, can explain the larger variance in MGT.

Result 5: Behavior in decomposed games is closer to resource allocation tasks with role certainty than to matrix games.

This result is consistent with both a inspection of the difference matrix in Table 2 and multidimensional results (explained below). RCT and MGT produce the largest difference between methods (0.741), and DGT and RUT produce the smallest difference (0.466). All of these differences are more that would be expected from chance alone. The test-retest reliability of the SVO Slider Measure is reported as 0.915 (Murphy et al, 2011), implying an expected difference of about

\footnotetext{
${ }^{3}$ For 9 subjects, who made hypothetical decisions in RCT, SVOs were exactly the same in RUT, DGT and MGT; for 7 subjects, who made incentivized decisions in RCT, SVOs were exactly the same in all four treatments.

${ }^{4}$ To check the robustness of results 1 to 4 we performed (bootstrapped) $t$-tests and excluded subjects who violated transitivity. The results are the same.
} 

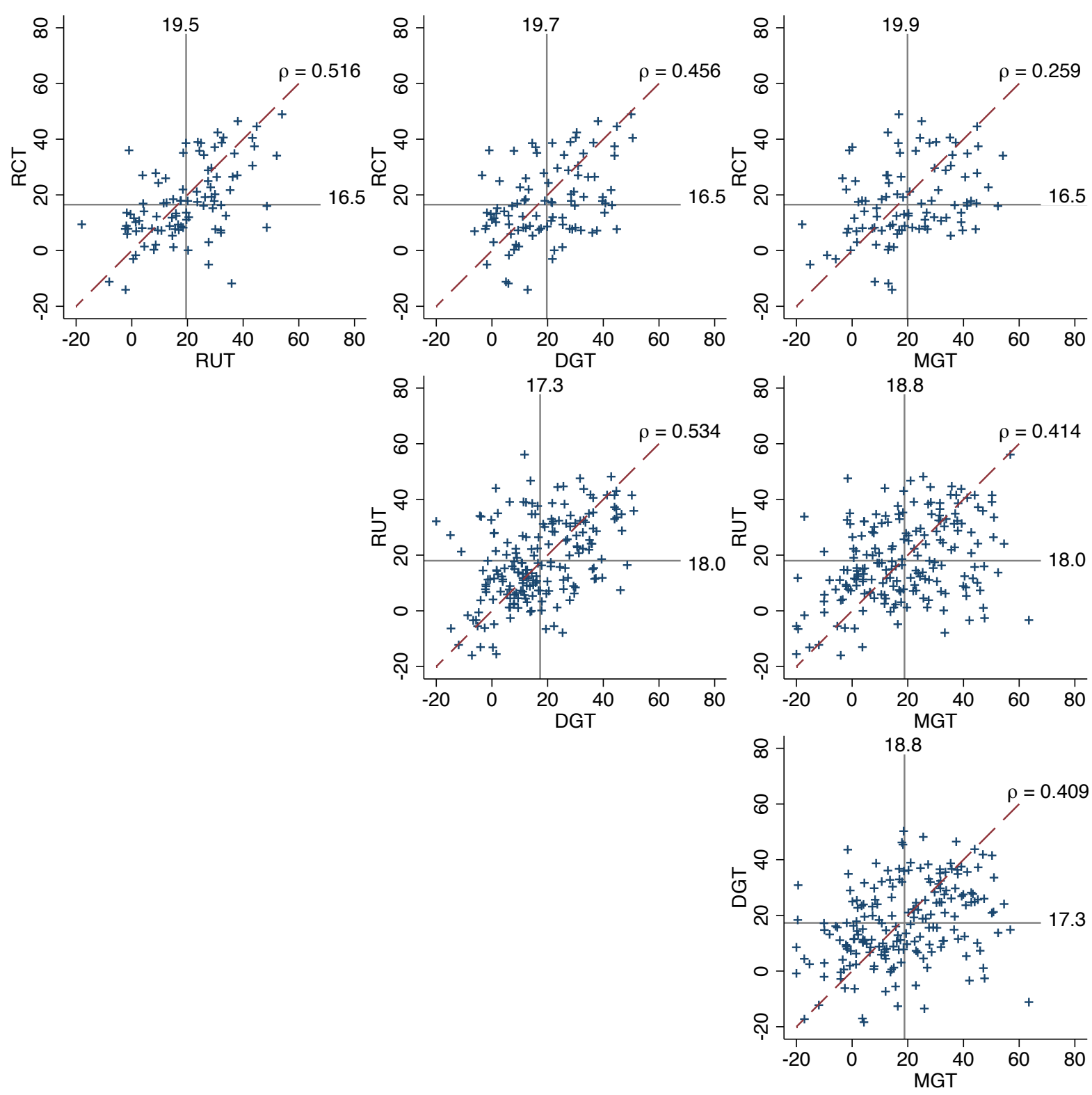

Figure 3: SVO scatterplots and Spearman's rank correlations $(r)$. Horizontal and vertical lines represent mean values of the corresponding treatment. (Some mean values differ from the mean values in Table 1 because in this figure, mean values are computed over all subjects who made incentivized decisions in both treatments, the treatment on the $x$-axis and the treatment on the $y$-axis.) 
0.085 (95\% CI 0.065 to 0.111 ) due to measurement error alone. The observed differences are substantially larger than this, indicating significant effects of social context on the measurement of distributive preferences at the individual level.

\begin{tabular}{c|cccc} 
& RCT & RUT & DGT & MGT \\
\hline RCT & 0 & - & - & - \\
RUT & 0.484 & 0 & - & - \\
DGT & 0.544 & 0.466 & 0 & - \\
MGT & 0.741 & 0.586 & 0.591 & 0
\end{tabular}

Table 2: Difference matrix among the different treatments. The entries are 1 minus the correlation between two methods. Higher numbers indicate more inconsistency in subject's behavior between two methods.

Results from multidimensional scaling yield the rank ordering of methods in terms of similarity. The resulting ordering is: RCT, RUT, DGT, and MGT. The output scores are $-0.4404,-0.1296$, $-0.0434,0.6135$ respectively. The stress of the model is $(1.65 \mathrm{e}-16)$ indicating adequacy of the goodness of fit of the unidimensional model. These multidimensional scaling scores can be normalized between 0 and 1 (see Figure 4), and the results show that behavior in DGT is closer to behavior in RCT than to MGT.

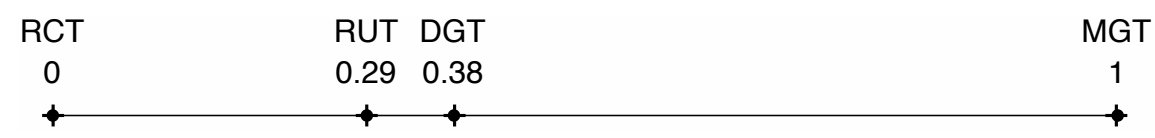

Figure 4: Normalized multidimensional scaling scores from each of the four treatments mapped onto a unidimensional solution. This analysis yields the relative similarity (behavioral consistency) among the different treatments.

Note that the large differences in scores between DGT and MGT is not inconsistent with Result 4. The central tendency of the DGT and MGT distributions are similar but that does not imply that individual behavior in the treatments is consistan. Rather we see that many DMs respond to the strategic framing, but in different ways.

\subsection{Implications for the measurement of distributional preferences}

In resource allocation tasks with role certainty strategic considerations are absent because the other subject affected by the DM's behavior is completely passive. Kerschbamer (2015b) argues that role certainty "seems to be the cleanest procedure from a theoretical point of view" (p. 1). We agree with this viewpoint and the results from RCT provide the most straightforward evidence about subject's distributive preferences.

We show evidence that different subjects respond differently as role uncertainty and strategic interdependence is introduced. This increased social interdependence may be of interest, but it creates complexity and creates multiple confounding factors (different behavioral forces triggered 
by social context) which make valid interpretations about revealed distributional preferences difficult. Because of this, our conclusions echo Kerschbamer who advocates measuring distributive preferences with role certainty.

We find no significant differences in average SVO given incentives. Nonetheless, we do not agree with Mentzakis and Mestelman (2013) who argue that eliciting SVO without financial incentives comes with no disadvantages. The difference in means, although not statistically significant, points to the presence of other factors, which may inflate SVO if no incentives are used. Moreover, hypothetical decisions inflate variance and reduce statistical power (see also Camerer and Hogarth, 1999).

When SVO is measured with role uncertainty, the resulting score should be taken as an upper bound to an individuals' distributional preferences. Role uncertainty is clearly a more efficient measurement method but it comes with trade-offs. Experimenters should weigh the benefits of the efficiency advantage with the possibility of inflating scores due to role uncertainty. Luckily for the efficiency-minded researcher the effect is not massive, and for work that only requires a rough approximation of distributive preferences the RUT approach may be sufficient.

This study is silent to which of these social contexts provides the best way to measure SVO. In order to establish evidence to address this question, one would have to measure SVO using a variety of different social contexts and then use these different individual scores to predict other behavior (predictive validity), correlate the scores on other measures (convergent validity), and show how the scores were separate from other constructs like beliefs (divergent validity). Ironically, a confounded measure of SVO (simultaneously measuring both preferences and beliefs) may make better predictions of cooperative choices rather than a pure measure of distributional preferences alone, as beliefs are also a good predictor of cooperative behavior. It is for this reason that one would have to measure beliefs in tandem with preferences, in order to establish the unique predictive capacity of SVO for cooperative behaviors. All of this is beyond the scope of the current paper, which had the modest goal of identifying to what degree, if at all, social contexts affect subject's SVO scores.

\section{Conclusion}

This article contributes to the literature on procedural differences and their effects on prosocial behavior. We systematically studied the effect of three different methods to measure SVO (RCT, RUT, DGT), and included an obvious strategic choice (MGT) setting. Although laboratory experiments offer a relatively clean environment, there remains at least some minimal social context in which the choices are embedded, as there is some "other" whose payoff is influenced by the choices of the DM. The role assignments create part of the social context, and we investigated how social context and its salience influences behavior. Overall results show that in the aggregate, distributional preferences are higher when assessed under role uncertainty or in decomposed games, compared to role certainty. Importantly, at the individual level, there are substantial non-systematic differences between treatments.

The comparison of decomposed games and matrix games adds to the literature on presentation effects (Charness et al, 2004; Requate and Waichman, 2010; Gürerk and Selten, 2011). The comparison of role assignment procedures also complements the studies by Murphy et al (2011) and Kerschbamer (2015a), which discuss how to identify distributional preferences based on sub- 
jects' choices in several resource allocation tasks but do not discuss the issue of role assignment. A better understanding of the influence of social context on behavior is desirable because it leads to a cleaner measurement of distributional preferences. From the perspective of a practitioner, who would like to control for subjects' preferences, a more precise measurement allows for better mitigation, which helps make predictions more effectively.

The overall pattern of results leads us to the following conclusions: Different people have distributional preferences to different degrees. We know from previous research that these preferences are relatively stable in the absence of information (i.e., in a test-retest sense). What we identify here is that these distributional preferences are sensitive to social contexts and increasing the social interdependence, and its salience, causes different people to respond in fundamentally different ways- some people become nicer, some become more selfish. We conjecture that the heterogeneity in responses to different social contexts is driven in large part by heterogeneous beliefs about what others are like. DMs homegrown preferences are made more salient by different social contexts, and the result is that DM's distributional preferences, as measured by the SVO Slider Measure, change in different ways as function of these beliefs. 


\section{References}

Andreoni J, Miller J (2002) Giving according to garp: An experimental test of the consistency of preferences for altruism. Econometrica 70(2):737-753

Balafoutas L, Kerschbamer R, Sutter M (2012) Distributional preferences and competitive behavior. Journal of Economic Behavior and Organization 83(1):125-135

Balafoutas L, Kerschbamer R, Kocher M, Sutter M (2014) Revealed distributional preferences: Individuals vs. teams. Journal of Economic Behavior and Organization 108:319-330, DOI 10. 1016/j.jebo.2013.12.012

Balliet D, Parks C, Joireman J (2009) Social value orientation and cooperation in social dilemmas: A meta-analysis. Group Processes \& Intergroup Relations 12(4):533-547, DOI \{10.1177/ $1368430209105040\}$

Bardsley N (2007) Dictator game giving: altruism or artefact? Experimental Economics 11(2):122-133

Blanco M, Engelmann D, Normann HT (2011) A within-subject analysis of other-regarding preferences. Games and Economic Behavior 72(2):321-338

Camerer CF, Fehr E (2004) Measuring social norms and preferences using experimental games: A guide for social scientists. In: Foundations of Human Sociality, Oxford University Press, pp $55-95$

Camerer CF, Hogarth RM (1999) The effects of financial incentives in experiments: A review and capital-labor-production framework. Journal of Risk and Uncertainty 19(1-3):7-42

Charness G, Grosskopf B (2001) Relative payoffs and happiness: An experimental study. Journal of Economic Behavior and Organization 45(3):301-328

Charness G, Frechette GR, Kagel JH (2004) How robust is laboratory gift exchange? Experimental Economics 7(2):189-205

Crosetto P, Weisel O, Winter F (2012) A flexible z-Tree implementation of the social value orientation slider measure (Murphy et al. 2011): Manual. Jena Economic Research Papers

Dana J, Weber RA, Kuang JX (2007) Exploiting moral wiggle room: experiments demonstrating an illusory preference for fairness. Economic Theory 33(1):67-80

Dariel A, Nikiforakis N (2014) Cooperators and reciprocators: A within-subject analysis of prosocial behavior. Economics Letters 122(2):163-166

Engelmann D, Strobel M (2004) Inequality aversion, efficiency, and maximin preferences in simple distribution experiments. The American Economic Review 94(4):857-869

Fischbacher U (2007) z-tree: Zurich toolbox for ready-made economic experiments. Experimental Economics 10(2):171-178 
Fisman R, Kariv S, Markovits D (2007) Individual Preferences for Giving. The American Economic Review 97(5):1858-1876

Forsythe R, Horowitz JL, Savin NE, Sefton M (1994) Fairness in simple bargaining experiments. Games and Economic Behavior 6(3):347-369

Gallo Jr PS, Funk SG, Levine JR (1969) Reward size, method of presentation, and number of alternatives in a prisoner's dilemma game. Journal of Personality and Social Psychology 13(3):239_ 244

Greiff M (2013) Affective social ties without the need to belong? Rationality, Markets and Morals $4: 20-27$

Gürerk Ö, Selten R (2011) The effect of payoff tables on experimental oligopoly behavior. Experimental Economics 15(3):499-509

Hillman AL (2010) Expressive behavior in economics and politics. European Journal of Political Economy 26(4):403-418

Iriberri N, Rey-Biel P (2011) The role of role uncertainty in modified dictator games. Experimental Economics 14(2):160-180

Kamecke U (1997) Rotations: Matching schemes that efficiently preserve the best reply structure of a one shot game. International Journal of Game Theory 26(3):409-417

Kerschbamer R (2013) The geometry of distributional preferences and a non-parametric identification approach. Working Papers in Economics and Statistics, University of Innsbruck

Kerschbamer R (2015a) The geometry of distributional preferences and a non-parametric identification approach - The equality equivalence test. European Economic Review 76(C):85-103

Kerschbamer R (2015b) The geometry of distributional preferences and a non-parametric identification approach: The equality equivalence test, Online Appendix. European Economic Review 76:1-9

Kirchgässner G (1992) Towards a theory of low-cost decisions. European Journal of Political Economy 8(2):305-320

van Lange PAM, Bekkers R, Schuyt TNM, van Vugt M (2007) From games to giving: Social value orientation predicts donations to noble causes. Basic and Applied Social Psychology 29(4):375384

Liebrand WBG (1984) The effect of social motives, communication and group size on behaviour in an N-person multi-stage mixed-motive game. European Journal of Social Psychology 14(3):239-264

Mentzakis E, Mestelman S (2013) Hypothetical bias in value orientations ring games. Economics Letters 120(3):562-565 
Messick DM, McClintock CG (1968) Motivational bases of choice in experimental games. Journal of Experimental Social Psychology 4(1):1-25

Murphy RO, Ackermann KA (2014) Social value orientation: Theoretical and measurement issues in the study of social preferences. Personality and Social Psychology Review 18(1):13-41

Murphy RO, Ackermann KA, Handgraaf MJJ (2011) Measuring social value orientation. Judgment and Decision Making 6(8):771-781

Offerman T, Sonnemans J, Schram A (1996) Value orientations, expectations and voluntary contributions in public goods. The Economic Journal 106(437):817-845

Pruitt DG (1967) Reward structure and cooperation: The decomposed prisoner's dilemma game. Journal of Personality and Social Psychology 7(1p1):21-27, DOI 10.1037/h0024914

Rabin M (1993) Incorporating fairness into game theory and economics. American Economic Review 83(5):1281-1302

Requate T, Waichman I (2010) "A profit table or a profit calculator?: A note on the design of Cournot oligopoly experiments. Experimental Economics 14(1):36-46

Rigdon ML, Levine AS (2009) The role of expectations and gender in altruism. MPRA Paper 19372 URL http: / /mpra.ub.uni-muenchen. de/19372/

Shafir E, Tversky A (1992) Thinking through uncertainty: Nonconsequential reasoning and choice. Cognitive psychology 24(4):449-474 


\section{Appendix}

\section{A Detailed experimental procedure}

The experiment was conducted at the University of Giessen using zTree (Fischbacher, 2007). A total of 192 subjects participated. Upon arrival, subjects were asked to take a seat at a randomly assigned computer workstation where they found general instructions which informed subjects that the experiment consists of four independent treatments. In addition to the general instructions, each subject found four sealed and differently colored envelopes on her desk, containing the instructions for the four different treatments.

Subjects were informed that at the beginning of each part, the on-screen instructions would inform them which colored envelope to open. This procedure ensures that subjects are informed about each treatment only when the treatment begins, but that the treatments exist a priori and are independent of their choices.

In order to avoid that subjects act prosocially in order to elicit kindness from reciprocators in subsequent tasks or treatments, subjects received feedback about results and earnings only at the very end of the experiment.

The order in which subjects encountered the four treatments was fully counterbalanced across sessions. That is, we implemented all permutations of the orders of treatments, resulting in $4 !=24$ orders that were each implemented in a separate session. The number of subjects per session was eight, and a no-contagion matching, which was also explained to subjects, was used (Kamecke, 1997).

At the beginning of each treatment, subjects opened the envelope containing the treatmentspecific instructions which were also read aloud by the experimenter, and subjects had the opportunity to ask questions privately. Before starting the experimental task subjects had to answer a control question correctly to verify their comprehension.

Each treatment consists of six different decision situations. In treatments RCT, RUT and DGT the six decision situations correspond to the six resource allocation tasks which comprise the six primary items of the SVO Slider Measure (see Figure 5). In treatment MGT the six decision situations correspond to six matrix games (see appendix $\mathrm{C}$ for all matrix games). ${ }^{5}$

Within each treatment, task order and option order of the six resource allocation tasks was fully randomized. These randomization procedures were implemented to control for order effects both within and across treatments, and moreover, to mitigate carryover effects from subjects remembering their previous choices in particular items and simply attempting to reproduce their former choice patterns rather than reflecting anew on their preferences in each task and treatment.

Subjects were informed that each subject's final payoff was given by the sum of each treatment's payoff plus a fixed show-up fee (5 euros). For each treatment, one of the six decision

\footnotetext{
${ }^{5}$ The resource allocation tasks were implemented in terms of zTree modules (Crosetto et al, 2012) that were slightly modified so that items consists of five options per task rather than nine options. We provide subjects with five options per task to ensure that the recomposition of the corresponding decomposed games into matrix games in normal form would not overwhelm subjects with too many strategies. In the most commonly used version, each item from the Slider Measure corresponds to the choice between nine alternatives. Recomposing these Slider Measure items would have resulted in a $9 \times 9$ payoff matrix with 81 cells. Reducing to 5 options yielded a matrix game that had only 25 cells which we considered experimentally tractable.
} 

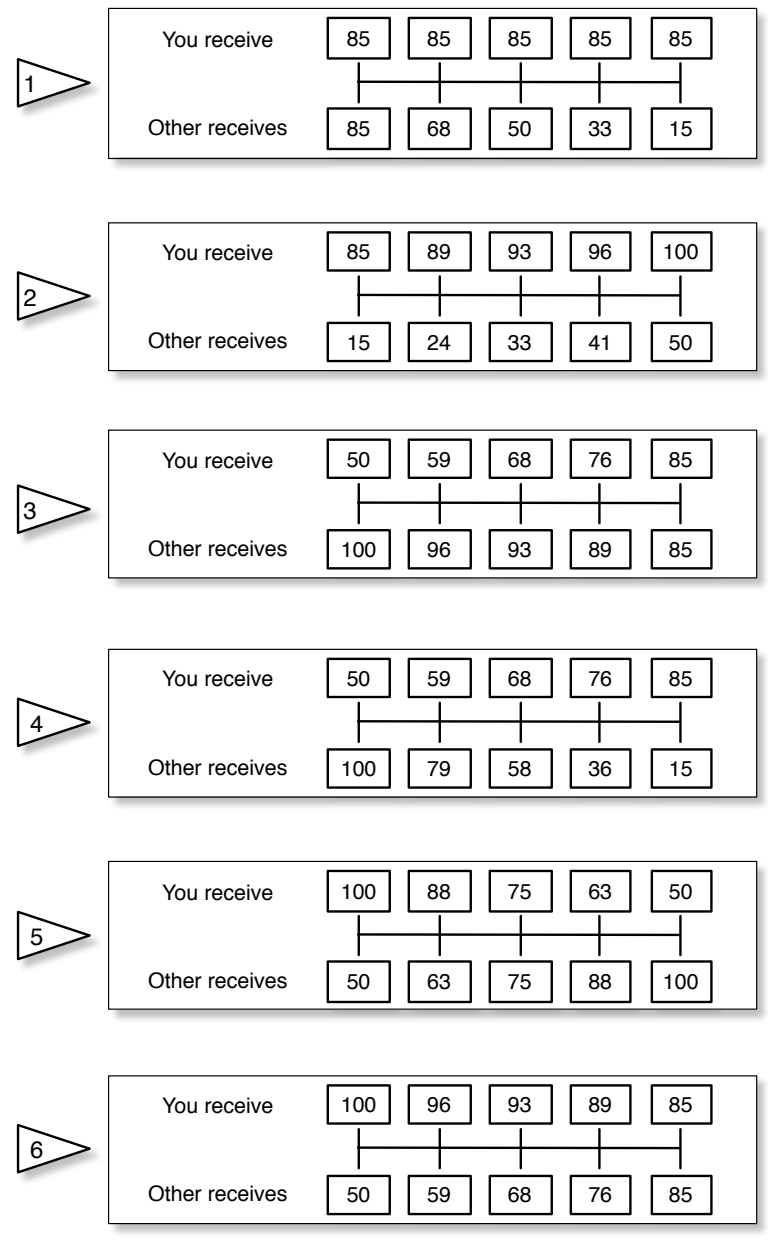

Figure 5: The six resource allocation tasks from the SVO Slider Measure. 
situations from each treatment was randomly selected, and the decisions made by both members in that decision situation determined payoffs from this treatment.

The experiment lasted 60 minutes and the average payment, including the show-up fee, was 14.55 Euros. 


\section{B Experimental Instructions}

Welcome to the experiment and thank you for your participation. Please read the instructions carefully. Do not talk to your neighbors during the entire experiment. If you have any questions please raise your hand. One of the experimenters will come to you and answer your questions in private. Following these rules is very important. Otherwise the results of this experiment will be scientifically worthless.

Please take your time reading the instructions and making your decisions. You are not able to influence the duration of the experiment by rushing through your decisions, because you always have to wait until the remaining participants have reached their decisions.

The experiment is completely anonymous. Neither during nor after the experiment you will be informed with whom you have interacted. No other participant will be informed about which role you were assigned to and how much you have earned. You will receive a show-up fee of 5 euros for your participation. Depending on your decisions and the decisions of the other participants you can additionally earn between 4 and 12 euros. You will be paid individually, privately, and in cash after the experiment. The expected duration of the experiment is 60 minutes. The exact course of the experiment will be described in the following.

The experiment consists of four parts which are independent from each other. Each part consists of six decision situations. The beginning of a new part of the experiment will be indicated on the screen. The instructions for each part of the experiment are in the colored envelopes. Please open the envelope with the relevant information only if the corresponding part of the experiment begins.

In each round, participants will be randomly matched in pairs, but you will never interact with the same participant twice. In other words, you can be sure that you will never interact with the same participant in several parts of the experiment. Furthermore, the matchings are done in such a way that the actions you take in one round cannot affect the actions of the people you will be paired with in later rounds. This also means that the actions of the participants you are paired with in a given round cannot be affected by your actions in earlier rounds. During the experiment, you will not receive any information about the decisions of the other participants, and other participants will not receive information about your decisions. During the experiment, the payoffs are denoted in points. The exchange rate between points and Euros is $1 / 50$. In other words, for 50 points you receive 1 Euro.

Calculation of your final payoff: After the experiment, a decision situation is randomly selected from each part of the experiment. Each decision situation has the same probability for being selected. That is, in every part each decision situation could be the decision situation, which determined your payoff from this part. Therefore, it makes sense to treat each decision situation as if it is the payoff-relevant decision situation.

The payoff that you get in addition to the 5 euros is the sum of the payoffs in the randomly selected decision situations. At the end of the experiment, you will be informed about the four randomly selected decision situations, the corresponding payoffs, and the sum of the payoffs. There will be a brief questionnaire after the last round is completed. After completion of the questionnaire, you will receive your payoff in cash. Payoffs will be made in private so that other participants won't be informed about your payoff.

The experiment will begin shortly. If you have any questions please raise your hand and wait until someone comes to your place. Please do not talk to the other participants during the entire 
experiment. Thank you for participating.

\section{Part "Red"}

In this part of the experiment, there are two roles, Decider, and Receiver, who will be randomly chosen. At the beginning, you will be informed about your role. In each of the six decision situation, the Decider chooses her most preferred allocation. That is, the decisions of the Decider determines her own payoff and the payoff of the Receiver. The Receiver has no influence on her own payoff and the payoff of the Decider. The Receiver chooses the allocation, that she would have chosen if she were in the role of the Decider. That is, that the decisions of the Receiver are purely hypothetical.

Your payoff from this part is calculated as follows: At the end of this part, a decision situation is randomly drawn and the allocation chosen by the Decider determines Decider's and Receiver's payoffs. You will be informed about the chosen decision situation and the corresponding payoffs at the end of the experiment.

\section{Part "Blue"}

In this part of the experiment, there will be six decisions. In each decision, you choose your most preferred allocation. The other participant faces the same decisions and has exactly same information as you. When you and the other participant have taken all decisions, it will randomly be determined whether payoffs are determined by your or the other participants' decisions.

Your payoff from this part is calculated as follows: At the end of this part, a decision situation is randomly drawn. In addition, a fair coin toss determines whether payoffs are determined by your or the other participants' decisions. You will be informed about the chosen decision situation and the corresponding payoffs at the end of the experiment.

\section{Part "Green"}

In this part of the experiment, there will be six decisions. In each decision, you choose your most preferred allocation. The other participant faces the same decisions and has exactly same information as you. The payoffs depend of your decisions and on the other participant's decision.

Your payoff from this part is calculated as follows: At the end of this part, a decision situation is randomly drawn. Your payoff is given by the amount you have allocated to yourself, and the amount that the other participant has allocated to you. The payoff of the other participant is given by the amount the other participant has allocated to herself, and the amount that you have allocated to the other participant. You will be informed about the chosen decision situation and the corresponding payoffs at the end of the experiment.

\section{Part "Yellow"}

In this part of the experiment, you interact with another participant in six decision situations. In each decision situation, both participants have to make a decision. That is, each participant chooses the option she prefers. The payoffs that are associated with each combination of decisions are displayed in the payoff table. 
In the row header there are the options that you can choose. In the column header there are the options that can be chosen by the other participant. The payoffs associated with a particular combination of choices are in the cell, in which the chosen row and column intersect. The entry to the left of the vertical bar within a cell is your payoff. The entry to the right of the vertical bar within a cell is the other participant's payoff.

Your payoff from this part is calculated as follows: A At the end of this part, a decision situation is randomly drawn. Your payoff and the other participant's payoff is determined by the options you and the other participant selected in this decision situations. You will be informed about the chosen decision situation and the corresponding payoffs at the end of the experiment. 


\section{The six matrix games used in MGT}

\begin{tabular}{|c|c|c|c|c|c|c|}
\hline & \multicolumn{5}{|c|}{ The other's options } \\
\hline & & Option A & Option B & Option C & Option D & Option E \\
\hline \multirow{5}{*}{ Your options } & Option A & 170,170 & 153,170 & 135,170 & 118,170 & 100,170 \\
\hline & Option B & 170,153 & 153,153 & 135,153 & 118,153 & 100,153 \\
\hline & Option C & 170,135 & 153,135 & 135,135 & 118,135 & 100,135 \\
\hline & Option D & 170,118 & 153,118 & 135,118 & 118,118 & 100,118 \\
\hline & Option E & 170,100 & 153,100 & 135,100 & 118,100 & 100,100 \\
\hline
\end{tabular}

Table 3: Recomposition of task number one into a matrix game.

\begin{tabular}{|c|c|c|c|c|c|c|}
\hline & \multicolumn{5}{|c|}{ The other's options } \\
\hline & & Option A & Option B & Option C & Option D & Option E \\
\hline \multirow{5}{*}{ Your options } & Option A & 100,100 & 109,104 & 118,108 & 126,111 & 135,115 \\
\hline & Option B & 104,109 & 113,113 & 122,117 & 130,120 & 139,124 \\
\hline & Option C & 108,118 & 117,122 & 126,126 & 134,129 & 143,133 \\
\hline & Option D & 111,126 & 120,130 & 129,134 & 137,137 & 146,141 \\
\hline & Option E & 115,135 & 124,139 & 133,143 & 141,146 & 150,150 \\
\hline
\end{tabular}

Table 4: Recomposition of task number two into a matrix game. 
The other's options

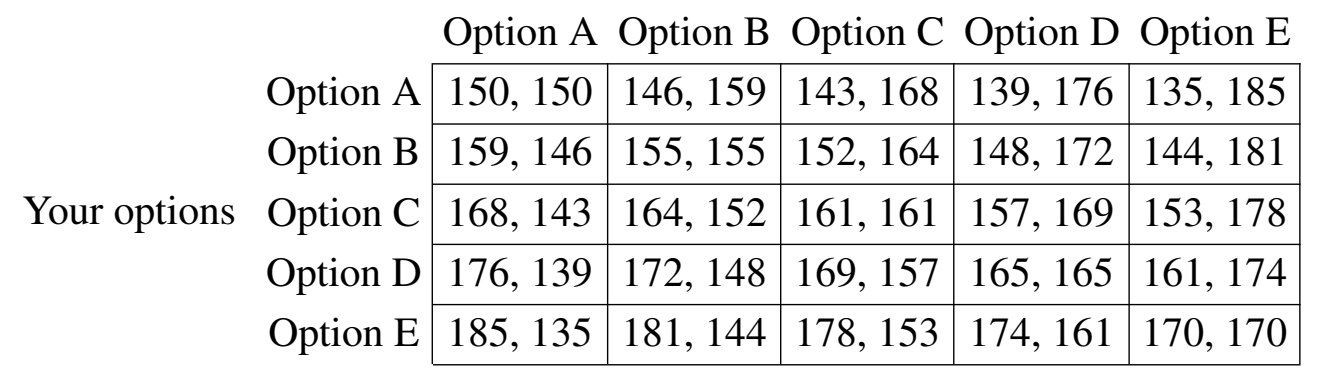

Table 5: Recomposition of task number three into a matrix game.

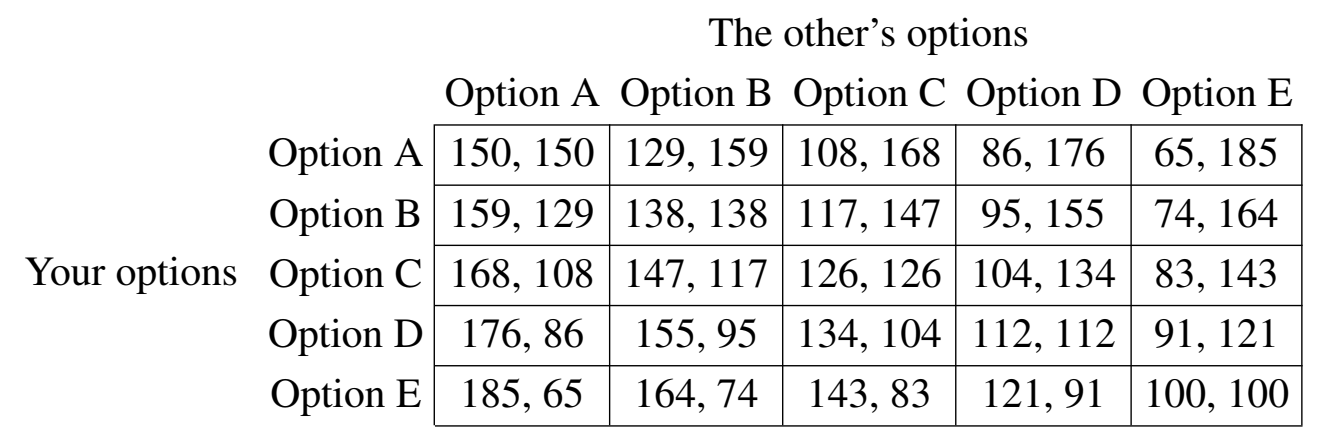

Table 6: Recomposition of task number four into a matrix game.

\begin{tabular}{|c|c|c|c|c|c|c|}
\hline & \multicolumn{5}{|c|}{ The other's options } \\
\hline & & Option A & Option B & Option C & Option D & Option E \\
\hline \multirow{5}{*}{ Your options } & Option A & 150,150 & 163,138 & 175,125 & 188,113 & 200,100 \\
\hline & Option B & 138,163 & 151,151 & 163,138 & 176,126 & 188,113 \\
\hline & Option C & 125,175 & 138,163 & 150,150 & 163,138 & 175,125 \\
\hline & Option D & 113,188 & 126,176 & 138,163 & 151,151 & 163,138 \\
\hline & Option E & 100,200 & 113,188 & 125,175 & 138,163 & 150,150 \\
\hline
\end{tabular}

Table 7: Recomposition of task number five into a matrix game.

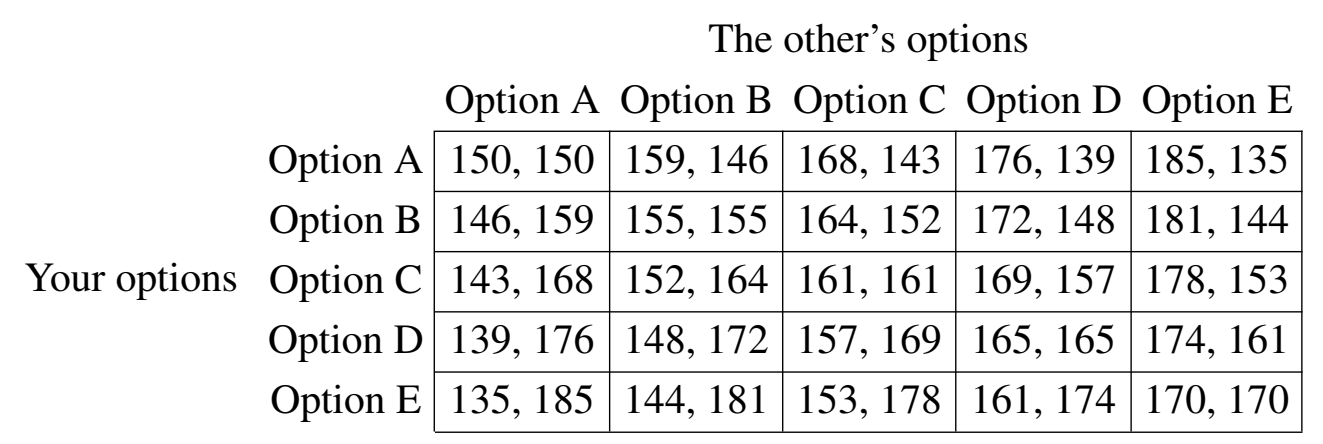

Table 8: Recomposition of task number six into a matrix game. 


\section{Histograms}
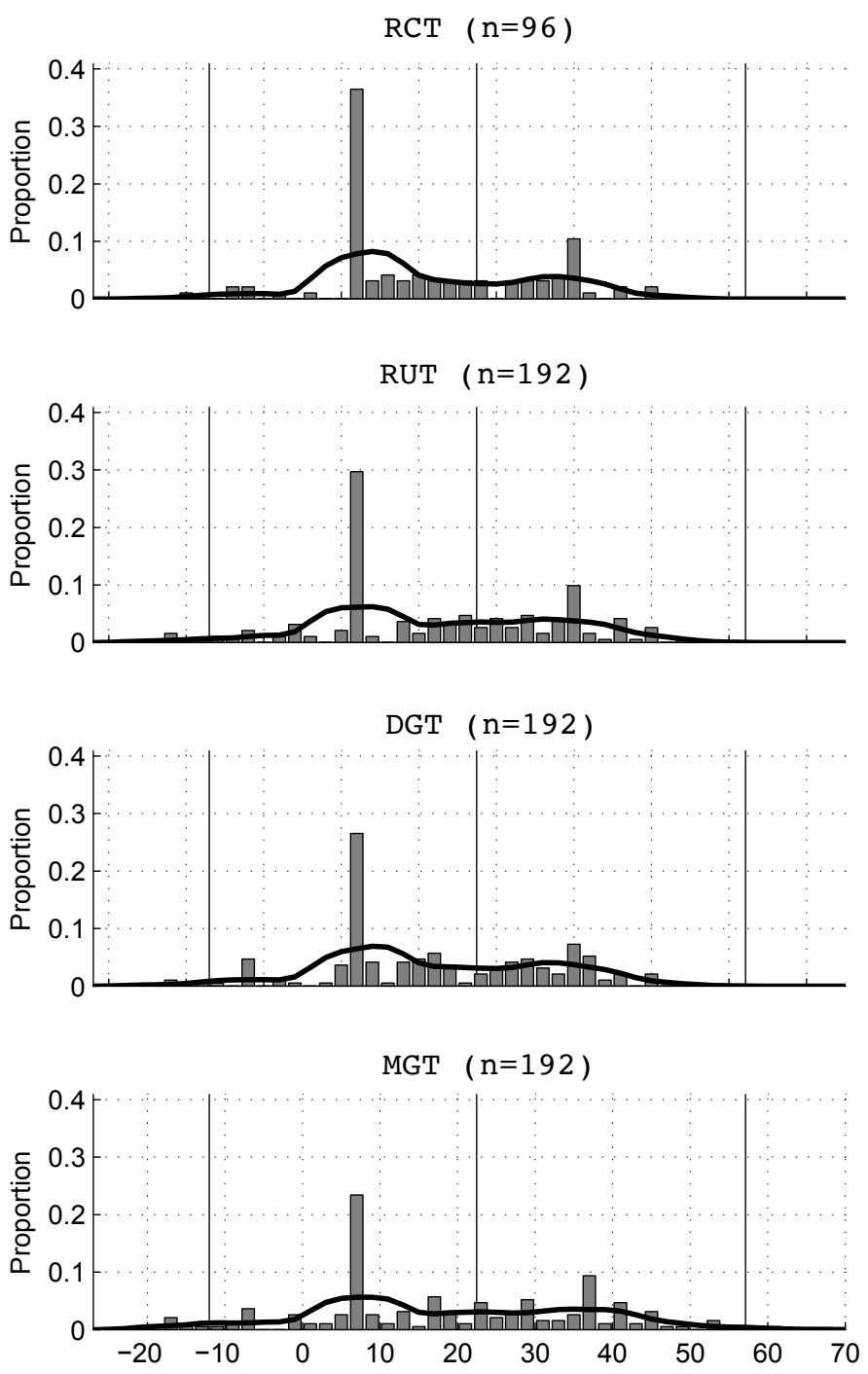

Figure 6: SVO Histograms. 


\section{E Tests for Order Effects}

In general, there is no systematic relation between SVO angles and the order of when they were assessed $(r=-0.03, p=0.33)$.
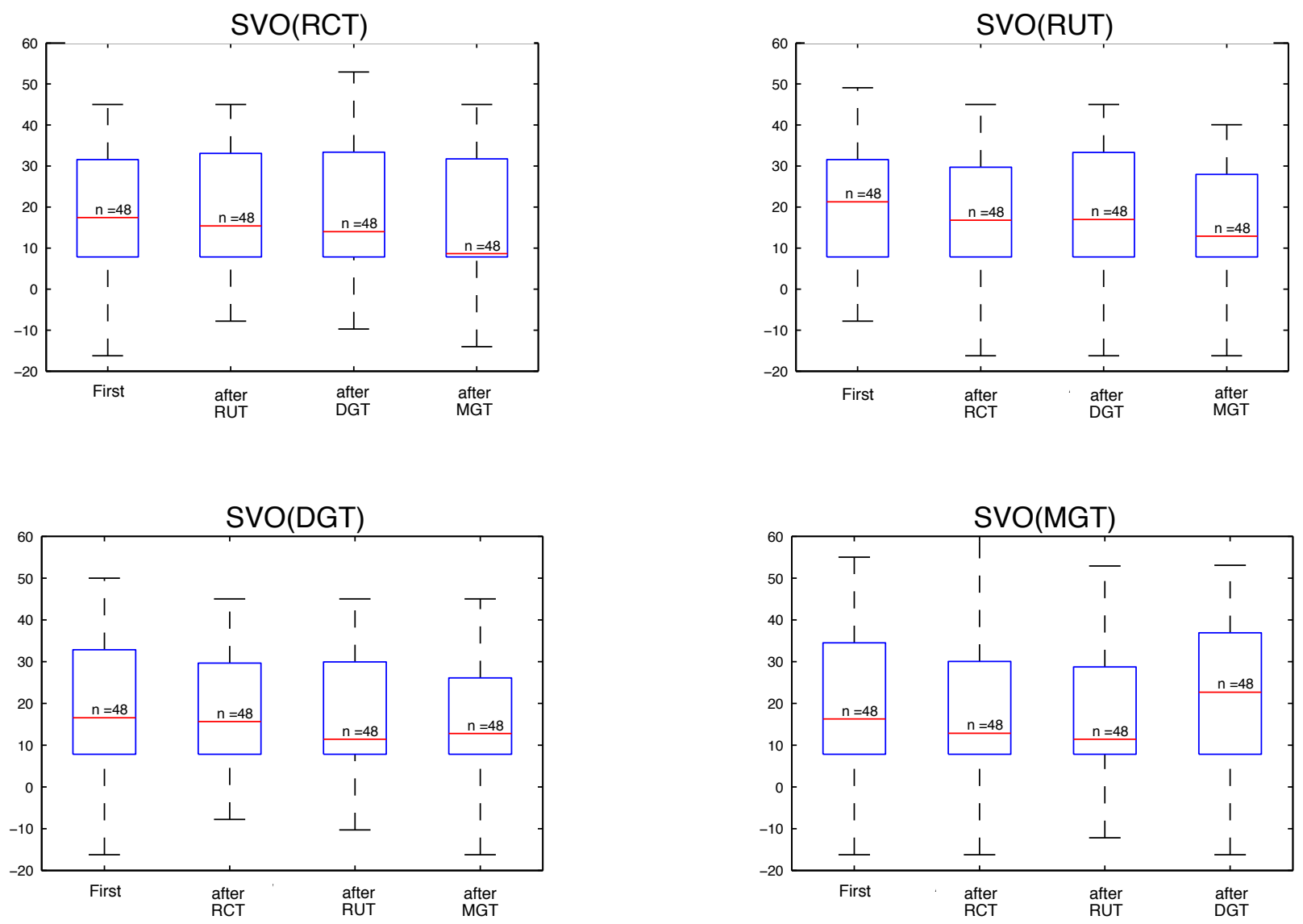

Figure 7: Descriptives of SVO angles per treatment depending on which other treatment was encountered before. 